\title{
Higher body fat percentage is associated with enhanced temperature perception in NAFLD: results from the randomised Wessex Evaluation of fatty Liver and Cardiovascular markers in NAFLD with OMacor thErapy trial (WELCOME) trial
}

\author{
Geraldine F. Clough $^{1}$ - Keith G. McCormick ${ }^{1}$ Eleonora Scorletti ${ }^{1,2}$ - Lokpal Bhatia $^{1,2}$. \\ Philip C. Calder $^{1,2}$ • Michael J. Griffin ${ }^{3}$. Christopher D. Byrne ${ }^{1,2}$
}

Received: 19 January 2016 / Accepted: 1 April 2016 /Published online: 22 April 2016

(C) Springer-Verlag Berlin Heidelberg 2016

\begin{abstract}
Aims/hypothesis The effect of $n-3$ fatty acid treatment on temperature perception as a sensory nerve function modality is uncertain. In patients with non-alcoholic fatty liver disease (NAFLD) both with and without type 2 diabetes, we: (1) tested whether 15-18 months' treatment with $4 \mathrm{~g}$ /day of docosahexaenoic plus eicosapentaenoic acid (DHA+EPA) improved hot (HPT) and cold (CPT) temperature perception thresholds and (2) explored factors associated with HPT and CPT, in a randomised, double-blind, placebo-controlled trial. Methods The effect of treatment $(n=44)$ on HPT, CPT and temperature perception index (TPI: difference between HPT and $\mathrm{CPT}$ ) was measured at the big toe in 90 individuals without neuropathy (type 2 diabetes; $n=30$ ). Participants were randomised 1:1, using sequential numbering, by personnel independent from the trial team. All participants and all members of the research team were blinded to group assignment.
\end{abstract}

Electronic supplementary material The online version of this article (doi:10.1007/s00125-016-3966-8) contains peer-reviewed but unedited supplementary material, which is available to authorised users.

Christopher D. Byrne

cdtb@soton.ac.uk

1 Human Development and Health Academic Unit, Faculty of Medicine, The Institute of Developmental Sciences (IDS), MP887, University of Southampton, Southampton General Hospital, Southampton SO16 6YD, UK

2 National Institute for Health Research Southampton Biomedical Research Centre, University of Southampton and University Hospital Southampton NHS Foundation Trust, Southampton, UK

3 Institute of Sound and Vibration Research, University of Southampton, Southampton, UK
Data were collected in the Southampton National Institute for Health Research Biomedical Research Centre. Treatment effects and the independence of associations were testing by regression modelling.

Results Mean \pm SD age was $50.9 \pm 10.6$ years. In men $(n=53)$ and women $(n=37)$, HPTs $\left({ }^{\circ} \mathrm{C}\right)$ were $46.1 \pm 5.1$ and $43.1 \pm 6.4$ $(p=0.02)$, CPTs $\left({ }^{\circ} \mathrm{C}\right)$ were $22.7 \pm 3.4$ and $24.5 \pm 3.6(p=0.07)$ and TPIs $\left({ }^{\circ} \mathrm{C}\right)$ were $23.4 \pm 7.4$ and $18.7 \pm 9.5(p=0.008)$, respectively. In univariate analyses, total body fat percentage (measured by dual-energy $\mathrm{x}$-ray absorptiometry [DXA]) was associated with HPT $(r=-0.36 p=0.001)$, CPT $(r=0.35 p=0.001)$ and TPI $(r=0.39 p=0.0001)$. In multivariable-adjusted regression models, adjusting for age, sex and other potential confounders, only body fat percentage was independently associated with HPT, CPT or TPI ( $p=0.006, p=0.006$ and $p=0.002$, respectively). DHA+EPA treatment did not modify HPT, CPT or TPI ( $p=0.93, p=0.44$ and $p=0.67$, respectively). There were no important adverse effects or side effects reported.

Conclusions/interpretation Higher body fat percentage is associated with enhanced temperature perception. There was no benefit of treatment with high-dose $n-3$ fatty acids on the thresholds to detect hot or cold stimuli.

Trial registration: ClinicalTrials.gov NCT00760513

Funding: This work was supported by the National Institute for Health Research through the NIHR Southampton Biomedical Research Unit grant and by a Diabetes UK allied health research training fellowship awarded to $\mathrm{KMcC}$ (Diabetes UK. BDA 09/0003937).

Keywords $n$-3 Fatty acid · Insulin resistance .

Microcirculation $\cdot$ Non-alcoholic fatty liver disease .

Omacor/Lovaza $\cdot$ Small sensory nerves .

Temperature perception threshold $\cdot$ Type 2 diabetes 


$\begin{array}{ll}\text { Abbreviations } & \\ \text { ACEI } & \text { ACE inhibitors } \\ \text { CPT } & \text { Cold temperature perception threshold } \\ \text { DHA } & \text { Docosahexaenoic acid } \\ \text { DXA } & \text { Dual energy x-ray absorptiometry } \\ \text { EPA } & \text { Eicosapentaenoic acid } \\ \text { HPT } & \text { Hot temperature perception threshold } \\ \text { MF } & \text { Maximum flux } \\ \text { MF/RF } & \text { The ratio of maximum flux (MF) to resting } \\ & \text { flux (RF) } \\ \text { NAFLD } & \text { Non-alcoholic fatty liver disease } \\ \text { QST } & \text { Quantitative sensory testing } \\ \text { RF } & \text { Resting flux } \\ \text { SFSN } & \text { Small sensory nerve fibre neuropathies } \\ \text { TPI } & \text { Temperature perception index } \\ \text { VPT } & \text { Vibration perception threshold } \\ \text { WELCOME } & \text { Wessex Evaluation of fatty Liver and } \\ & \text { Cardiovascular markers in non-alcoholic fatty } \\ & \text { liver disease (NAFLD) with OMacor thErapy }\end{array}$

\section{Introduction}

Small sensory nerve fibre neuropathies (SFSN) give rise to the symptoms of impaired nerve sensitivity and neuropathic pain which affect over $90 \%$ of people with type 1 and type 2 diabetes $[1,2]$ and are the main initiating factors for foot ulceration and lower limb amputation in people with diabetes [3]. The mechanisms underlying SFSN and neuropathic pain are not well understood but may include metabolic factors such as elevated intracellular glucose levels in both vascular and neural tissues, which can lead to increased oxidative stress and/or microvascular impairment and reduced nerve blood flow [4, 5].

Quantitative sensory testing (QST) is a method for assessing the somatosensory nervous system and is routinely used in the clinical assessment of large and small sensory nerve fibre function $[6,7]$. We have recently shown using QST of large myelinated nerve function that both ageing and a measure of microvascular reactivity were independently associated with altered vibration perception threshold (VPT) at $125 \mathrm{~Hz}$ in a high-risk patient group without evidence of overt peripheral neuropathy [8]. QST has also been used to study functional deficits in small fibre neuropathies, with temperature perception thresholds being one of the more widely employed tests [9-11]. Such tests provide an indirect but reproducible assessment of human small nerve function [12, 13].

There are as yet no treatments that prevent or reverse painful diabetic neuropathy. Vasodilator sprays may improve nerve blood flow [14] and alpha lipoic acid [15] (as a sulphur-containing product derived from the eight-carbonatom fatty acid octanoic acid) has been shown to give moderate pain relief in clinical trials, with fewer side effects than opioids and antidepressants [16]. Treatment with highdose long-chain polyunsaturated $n-3$ fatty acids such as eicosapentaenoic acid (EPA, 20:5 n-3) and docosahexaenoic acid (DHA, 22:6 n-3) has been shown to improve vibration perception and clinical symptoms of neuropathy in patients with type 2 diabetes [17] and to improve endothelial function in dyslipidaemia, overweight men [18], as well as offering neuroprotection by increasing nerve blood flow in an experimental model of diabetic neuropathy [19]. However, the benefit of long-chain $n-3$ fatty acids in patients at risk of, but without peripheral neuropathy, remains uncertain.

In a pre-specified sub-study of the Wessex Evaluation of fatty Liver and Cardiovascular markers in NAFLD with OMacor thErapy (WELCOME) trial (ClinicalTrial.gov registration no. NCT00760513) [20, 21] (a randomised doubleblind placebo-controlled trial), we tested the effect of DHA+ EPA (Omacor/Lovaza) treatment on hot and cold temperature perception thresholds. Our overall aim was to test the hypothesis that the highest licensed dose of DHA+EPA $n-3$ fatty acid treatment ( $4 \mathrm{~g} /$ day as ethyl esters) over a minimum of 15 months and a maximum of 18 months had beneficial effects on small sensory nerve function in high-risk patients for neuropathy, who had insulin resistance and non-alcoholic fatty liver disease (NAFLD) (with or without type 2 diabetes).

\section{Methods}

\section{Study design}

The participants formed part of the WELCOME study and the investigations were approved by the local research ethics committee (REC: 08/H0502/165) [20, 21]. One hundred and five individuals were recruited to the study (see Electronic Supplementary Material [ESM] Fig. 1 for the consort diagram and testing the effect of the intervention on this secondary endpoint). Participants were excluded from the current analysis if they had evidence of distal peripheral neuropathy in their feet, as suggested by their failure to detect a $10 \mathrm{~g}$ monofilament [22] $(n=4)$, or if they had evidence of diabetic eye disease at retinal screening $(n=0)$. Baseline and end of study thermal thresholds were obtained on 90 individuals (53 men and 37 women; mean age of $50.9 \pm 10.6$ years) of whom 44 were on active treatment and 46 on placebo. Participants gave their informed written consent. Biochemical and anthropometric measurements have been described previously [8].

\section{Peripheral neurological and microvascular function testing}

All tests were conducted in a temperature controlled room $\left(22-24^{\circ} \mathrm{C}\right)$ as described previously [8]. Participants refrained 
from caffeine-containing drinks, smoking and exercise for $\geq 2 \mathrm{~h}$ prior to testing. Measurements were made at baseline and end of study.

Temperature perception testing Hot (HPT) and cold (CPT) temperature perception thresholds were measured on the pulp of the left big toe using a thermal aesthesiometer (HVLab Diagnostics Instruments, Southampton, UK) [23] (see ESM Methods for details). A threshold temperature perception 'index' (TPI) was constructed for each individual, calculated as the arithmetic difference between HPT and CPT $\left({ }^{\circ} \mathrm{C}\right)$ measured at baseline [24].

Vibrotactile perception testing VPTs at $125 \mathrm{~Hz}$ were measured on the pulp of the left big toe as described previously [8] (see ESM Methods for details).

Microvascular function Cutaneous microvascular blood flow was assessed on the ventral surface of the nondominant forearm using laser Doppler fluximetry (MoorVMS LDF2, Moor Instruments, Axminster, UK) at rest (RF) and during the dilator response to transient ischaemia (180 mmHg for $3 \mathrm{~min}$; maximum flux [MF]) [8] (see ESM Methods for details).

\section{Statistical analysis}

Statistical analyses were undertaken using IBM SPSS Statistics 21.0 (IBM UK). Pearson and Spearman rank correlation coefficients were used to investigate associations between normally and non-normally distributed variables, respectively. In all cases a value of $p<0.05$ was taken to indicate significance. Multivariable linear regression modelling was used to test the independence of associations between baseline variables and baseline measurements of HPT, CPT and TPI. Explanatory factors that were included in the regression models included age, sex, diabetes (yes/no) (or HOMA-IR), measures of body fat and MF/RF. In additional analyses we also tested for effects of medications and skin temperature. We also tested the effects of DHA+EPA treatment on TPI and both HPT and CPT using multivariable linear regression modelling. For all three outcomes of interest the outcome represented the 'change' variable, i.e. the difference between the end of study measurement and the baseline study measurement. For each separate regression model, there was also adjustment for the baseline measurement of the respective outcome variable in question. Each of these models included DHA+EPA treatment as a dichotomous exposure variable age, sex, the temperature threshold variable at baseline (of the respective outcome variable) and additional relevant exposures of interest, selected from the univariable analyses (including total body fat $\%, \mathrm{MF} / \mathrm{RF}$, and diabetes status (yes/no) or insulin resistance (HOMA-IR)).

\section{Results}

\section{Characteristics of the group}

Table 1 shows the baseline characteristics of the participants without overt neuropathy or microvascular disease. Of the participants, 35 were treated with statins, 32 with antihypertensive drugs, seven with fibrates, 18 with ACE inhibitors (ACEI), 24 with metformin and 13 with antidepressants.

\section{Effect of the $\boldsymbol{n}-\mathbf{3}$ polyunsaturated fatty acid intervention on temperature perception thresholds}

Individual baseline and end of study measurements for HPT, CPT and TPI for participants are shown in Fig. 1. Table 2 shows the results of multivariable regression modelling testing the effects of the DHA+EPA intervention on the change in HPT, CPT and TPI (i.e. the change between the end and start of the study) and shows no effect of the treatment on each of the outcomes of interest. Swapping HOMA-IR for diabetes status had little effect on the models.

\section{Associations between HPT and CPT and cardiovascular and metabolic risk factors at baseline}

In univariate analyses (see ESM for table of univariate analyses showing associations between hot and cold temperature perception thresholds and cardiovascular and metabolic risk factors at baseline), both HPT and CPT were associated with sex $(r=-0.252 p=0.017$ and $r=0.244$ $p=0.021$, respectively), with women being more sensitive to the thermal stimuli than men. HPTs and CPTs were associated with total body fat percentage and subcutaneous abdominal fat quantity. The associations between HPT and CPT measured at the big toe and percentage body fat were independent of skin temperature (mean skin temperature measured at the big toe prior to testing was $\left.28.2 \pm 2.5^{\circ} \mathrm{C}(n=90)\right)$ and were not affected by adjustment for skin temperature. Scatter plots for the association between HPT and CPT and body fat are shown in Fig. 2. The direction of the correlations shows that temperature perception (or the ability to detect the stimulus after a small temperature change) increases with increasing total body fat percentage. TPI showed a similar association with total body fat percentage $(r=0.39, p=0.0001)$ (Fig. 2) with the direction of correlation showing a reduction in TPI with increasing body fat. Similar trends were seen at the left middle finger (data not shown).

There was no significant association between HPT and $\mathrm{CPT}$ and measures of glycaemic status (fasting glucose concentration or $\mathrm{HbA}_{1 \mathrm{c}}$, participants with or without diabetes) or insulin resistance (HOMA-IR, participants without diabetes). In individuals with diabetes $(n=30)$, HPT was associated with 
Table 1 Baseline characteristics according to diabetes status and randomisation group

\begin{tabular}{|c|c|c|c|c|c|c|}
\hline Variable & $\begin{array}{l}\text { No diabetes } \\
n=60\end{array}$ & $\begin{array}{l}\text { Diabetes } \\
n=30\end{array}$ & $p$ value & $\begin{array}{l}\text { Randomised to placebo } \\
n=46\end{array}$ & $\begin{array}{l}\text { Randomised to DHA+EPA } \\
n=44\end{array}$ & $p$ value \\
\hline $\operatorname{Sex}(M / F)$ & $35 / 25$ & $18 / 12$ & & $31 / 15$ & $22 / 22$ & \\
\hline Age (years) & $51.5 \pm 10.7$ & $49.8 \pm 10.6$ & 0.69 & $54.1 \pm 9.6$ & $47.6 \pm 10.7$ & 0.003 \\
\hline BMI $\left(\mathrm{kg} / \mathrm{m}^{2}\right)$ & $32.1(4.8)$ & $33.4(6.0)$ & 0.03 & $31.9(5.2)$ & $32.4(7.1)$ & 0.09 \\
\hline Duration of diabetes (years) & & $3(3.5)$ & & $6(5)$ & $3(5)$ & 0.45 \\
\hline Diabetes & & & & 16 & 14 & \\
\hline Current smoker & 6 & 3 & 0.75 & 4 & 5 & 0.71 \\
\hline 10-year cardiovascular disease risk (QRISK2, \%) & $9.7(7.5)$ & $14.7(9.4)$ & 0.02 & $12.5(8.3)$ & $7.5(13.4)$ & 0.15 \\
\hline Systolic BP (mmHg) & $137(16)$ & $135(14)$ & 0.61 & 137 (17) & $135(26)$ & 1.0 \\
\hline Cholesterol (mmol/l) & $4.8(1.4)$ & $4.3(0.9)$ & 0.01 & $4.5(1.4)$ & $4.8(1.3)$ & 0.06 \\
\hline LDL-cholesterol (mmol/l) & $2.9(0.9)$ & $2.6(0.9)$ & 0.16 & $2.7(1.0)$ & $3.0(1.3)$ & 0.14 \\
\hline HDL-cholesterol (mmol/l) & $1.1(0.4)$ & $1.0(0.2)$ & 0.15 & $1.1(0.3)$ & $1.0(0.4)$ & 0.17 \\
\hline Cholesterol/HDL-cholesterol & $5.0 \pm 1.5$ & $4.7 \pm 1.5$ & 0.49 & $4.5 \pm 1.4$ & $5.3 \pm 1.6$ & 0.11 \\
\hline Triacylglycerol (mmol/l) & $1.8(1.3)$ & $1.5(1.0)$ & 0.56 & $1.5(1.0)$ & $1.8(1.2)$ & 0.55 \\
\hline Total body fat (DXA, \%) & $37.7 \pm 7.5$ & $36.8 \pm 7.0$ & 0.57 & $35.6 \pm 6.9$ & $39.4 \pm 7.1$ & 0.38 \\
\hline Subcutaneous fat (MRI, \%) & $32.4 \pm 9.9$ & $31.5 \pm 10.7$ & 0.68 & $29.9 \pm 9.4$ & $34.4 \pm 10.2$ & 0.14 \\
\hline Visceral fat (MRI, \%) & $16.0 \pm 4.8$ & $16.3 \pm 5.2$ & 0.83 & $16.8 \pm 4.6$ & $15.4 \pm 5.2$ & 0.30 \\
\hline Liver fat (MRS, \%) & $23.3(21.8)$ & $21.8(14.9)$ & 0.95 & $21.7(20.1)$ & $23.0(33.9)$ & 0.44 \\
\hline NAFLD fibrosis score ${ }^{\mathrm{a}}$ & $8.7(0.7)$ & $9.0(0.9)$ & 0.05 & $8.9(0.9)$ & $8.7(1.0)$ & 0.42 \\
\hline Insulin resistance (HOMA-IR) & $2.7(2.5)$ & $4.3(4.1)$ & 0.01 & $2.8(3.2)$ & $2.9(3.2)$ & 0.60 \\
\hline $\mathrm{HbA}_{1 \mathrm{c}}(\%)$ & $5.8(0.6)$ & $7.7(1.8)$ & 0.03 & $6.1(1.6)$ & $5.9(1.2)$ & 0.13 \\
\hline $\mathrm{HbA}_{1 \mathrm{c}}(\mathrm{mmol} / \mathrm{mol})$ & $42.8(4.4)$ & $56.9(13.3)$ & & $45.0(11.8)$ & $43.6(8.9)$ & \\
\hline Microvascular reactivity (MF/RF) & $4.2(1.5)$ & $5.0(1.8)$ & 0.33 & $4.3(2.0)$ & $4.3(2.4)$ & 1.0 \\
\hline Vibration threshold $(125 \mathrm{~Hz})\left(\mathrm{m} / \mathrm{s}^{2}\right)$ & $3.7(5.6)$ & $3.6(6.7)$ & 0.48 & $5.5(6.5)$ & $2.9(4.0)$ & 0.03 \\
\hline \multicolumn{7}{|l|}{ Temperature perception threshold $\left({ }^{\circ} \mathrm{C}\right)$} \\
\hline Hot & $45.4(6.0)$ & $46.7(5.6)$ & 0.76 & $45.4(5.8)$ & $43.8(5.7)$ & 0.19 \\
\hline Cold & $23.0(3.6)$ & $24.4(3.4)$ & 0.38 & $22.6(3.5)$ & $24.8(3.9)$ & 0.08 \\
\hline
\end{tabular}

Data are presented as mean $\pm \mathrm{SD}$ or median (interquartile range)

${ }^{a}$ NAFLD fibrosis score, see reference [36]

the duration of diabetes $(r=0.471, p=0.009)$, but CPT was $\operatorname{not}(r=-0.267, p=0.154)$.

HPT was associated with CPT $(r=-0.670, p<0.0001)$, a finding consistent with that in a cohort of 300 healthy students [25]. Temperature perception was also associated with VPT measured at $125 \mathrm{~Hz}$ at the big toe [8] (HPT $r=0.326$, $p=0.002$; CPT $r=-0.211, p=0.046$ ).

Using regression modelling, we explored the independence of associations between HPT, CPT and TPI and each of the variables that were shown to be associated with these outcomes in the univariate analyses. HPT, CPT and TPI were the respective outcomes in these separate regression models (Table 3). These multivariable models showed that the exposure variables in the models explained $21 \%$ of the variance in HPT, $18 \%$ in CPT and $23 \%$ of the variance in TPI. Of note, in each of these regression models there was a strong independent association between total body fat percentage and HPT, CPT and TPI. Swapping HOMA-IR for diabetes status had little effect on each regression model (data not shown). Use of statins, fibrates, metformin, antidepressants or all antihypertensive drugs (including calcium antagonists and ACEIs) was not independently associated with thermal perception thresholds when each factor was added to the model as a dichotomous exposure variable (data not shown).

\section{Discussion}

Our novel results show no benefit from DHA+EPA treatment on hot or cold temperature perception thresholds, in patients with NAFLD, both with and without diabetes. We have examined the effects of the highest licensed dose of $n-3$ fatty acids ( $4 \mathrm{~g}$ /day as Omacor/Lovaza) on measures of small sensory nerve fibre function in a randomised double-blind placebo-controlled trial lasting a minimum of 15 months and a maximum of 18 months in participants at risk of developing diabetic neuropathy and neuropathic pain. To our knowledge, our study is the first randomised double-blind placebo- 

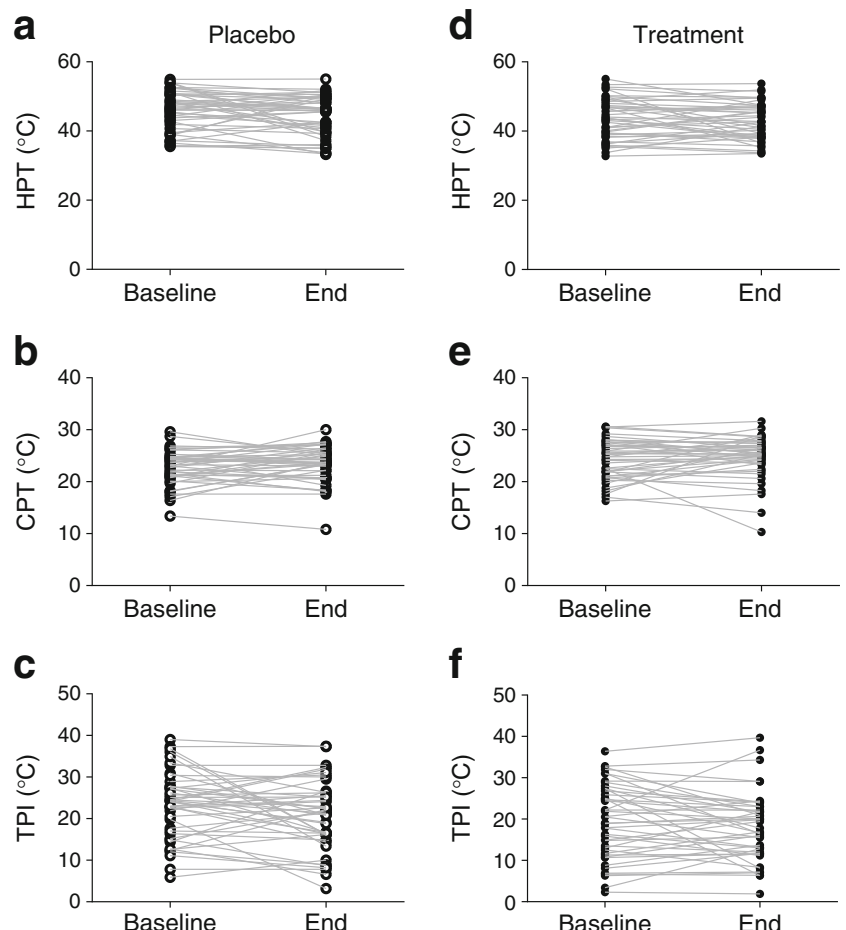

Fig. 1 Effect of 15-18 months' treatment with DHA+EPA on HPT, CPT and TPI (difference between HPT and CPT) measured at the big toe. (a-c) the placebo group; $(\mathbf{d}-\mathbf{f})$ the treatment group

controlled trial to examine the effects of DHA+EPA on temperature perception. We also show for the first time that hot and cold thermal perception thresholds were strongly and independently associated with total body fat percentage (but not with liver fat or with glycaemic control) in men and women.
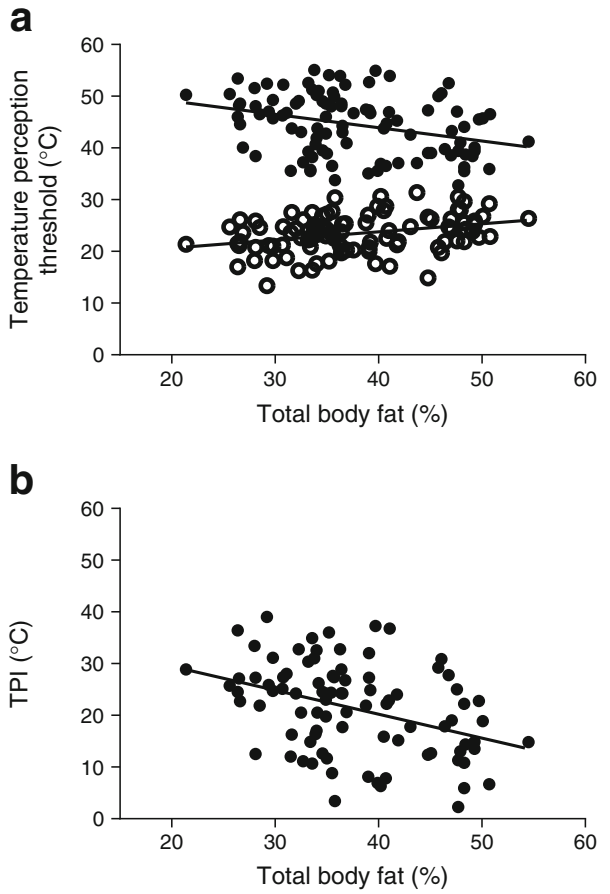

Fig. 2 Scatter plots showing the relationship between (a) baseline HPT and CPT and (b) TPI (difference between HPT and CPT) measured at the big toe and total body fat percentage measured using DXA

We found no association between hot or cold temperature perception thresholds and measures of insulin sensitivity or $\mathrm{HbA}_{1 \mathrm{c}}$ in these patients who were at risk of neuropathy. Our findings are consistent with the results of a study of 156 individuals with peripheral neuropathy where there was no association between neuropathy and glycaemic control [26].
Table 2 Multivariable regression models showing the effects of DHA+EPA treatment on HPT and $\mathrm{CPT}$, and on the TPI, adjusting for baseline measurement of each respective outcome, and key covariates and potential confounders as explanatory variables

\begin{tabular}{llll}
\hline Independent variable & \multicolumn{2}{l}{ Primary outcome ${ }^{\mathrm{a}}$} & \\
\cline { 2 - 4 } & $\mathrm{TPI}\left({ }^{\circ} \mathrm{C}\right)$ & $\mathrm{HPT}\left({ }^{\circ} \mathrm{C}\right)$ & $\mathrm{CPT}\left({ }^{\circ} \mathrm{C}\right)$ \\
\hline Treatment DHA+EPA & $-0.660(-3.680,2.359)$ & $-0.091(-2.25,2.07)$ & $0.575(-0.88,2.03)$ \\
& 0.665 & 0.93 & 0.44 \\
Age (years) & $-0.014(-0.159,0.130)$ & $0.045(-0.059,0.148)$ & $0.053(-0.02,0.12)$ \\
& 0.845 & 0.39 & 0.13 \\
Male sex & $3.122(-1.823,8.068)$ & $1.31(-2.22,4.85)$ & $-1.91(-4.27,0.46)$ \\
& 0.213 & 0.46 & 0.11 \\
Diabetes status (yes/no) & $0.007(-2.909,2.924)$ & $-0.041(-2.13,2.05)$ & $-0.097(-1.51,1.31)$ \\
& 0.996 & 0.969 & 0.89 \\
Temperature perception & $-0.392(-0.573,-0.212)$ & $-0.451(-0.64,-0.26)$ & $-0.391(-0.60,-0.19)$ \\
threshold/index at baseline & $<0.0001$ & $<0.0001$ & $<0.0001$ \\
MF/RF & $-0.178(-1.113,0.758)$ & $-0.213(-0.88,0.46)$ & $0.013(-0.43,0.46)$ \\
& 0.707 & 0.53 & 0.057 \\
Total body fat DXA (\%) & $-0.167(-0.519,0.185)$ & $-0.077(-0.33,0.17)$ & $0.11(-0.06,0.28)$ \\
& 0.348 & 0.54 & 0.19 \\
\hline
\end{tabular}

Data are presented as unstandardised B coefficient $(95 \% \mathrm{CI})$ with $p$ values shown below

${ }^{a}$ For all primary outcomes, data are given as change from baseline to end of study 
Table 3 Multivariable regression models with HPT, CPT and TPI as the outcomes, and key covariates and potential confounders as explanatory variables

\begin{tabular}{llll}
\hline Independent variable & \multicolumn{2}{l}{ Primary outcome } & \\
\cline { 2 - 4 } & TPI $\left({ }^{\circ} \mathrm{C}\right)$ at baseline & HPT $\left({ }^{\circ} \mathrm{C}\right)$ at baseline & CPT $\left({ }^{\circ} \mathrm{C}\right)$ at baseline \\
\hline Age (years) & $0.129(-0.033,0.291)$ & $0.086(-0.024,0.197)$ & $-0.043(-0.112,0.026)$ \\
& 0.116 & 0.125 & 0.218 \\
Male sex & $2.7(-3.5218,8.619)$ & $1.671(-2.376,5.718)$ & $-1.030(-3.552,1.452)$ \\
& 0.367 & 0.414 & 0.419 \\
MF/RF & $-1.04(-2.141,0.060)$ & $-0.775(-1.527,-0.022)$ & $0.266(-0.203,0.735)$ \\
& 0.064 & 0.044 & 0.263 \\
Diabetes status (yes/no $)$ & $-0.234(-3.739,3.270)$ & $0.497(-1.899,2.893)$ & $0.731(-0.762,2.225)$ \\
& 0.895 & 0.681 & 0.333 \\
Total body fat DEXA $(\%)$ & $-0.622(-1.016,-0.227)$ & $-0.383(-0.653,-0.113)$ & $0.238(0.070,0.406)$ \\
& 0.002 & 0.006 & 0.006
\end{tabular}

Data are presented as unstandardised B coefficient $(95 \% \mathrm{CI})$ with $p$ values given underneath

Multivariable regression models for all participants completing the randomised double-blind placebo-controlled trial testing the effect of DHA+EPA treatment on each of the primary outcomes. Placebo group, $n=46$; treatment group, $n=44$. Each regression model was adjusted for age, sex, outcome variable value at baseline, plus diabetes status (yes/no), a measure of microvascular dilator capacity (MF/RF) and total body fat $\%$ (DXA). For the models: TPI: $R^{2}=0.231$; adjusted $R^{2}=0.186(p=0.001)$. HPT $R^{2}=0.21$; adjusted $R^{2}=0.166(p=0.001)$. CPT $R^{2}=0.182 ;$ adjusted $R^{2}=0.133(p=0.004)$
Elevated hot thresholds have been shown to be the most frequent sensory deficit in individuals with diabetes and hot thresholds have been shown to be correlated with glycaemic control in some [9, 27] but not all [28, 29] studies. In those individuals with diabetes, HPT was associated with duration of diabetes, possibly due to increased oxidative stress, nerve damage or reducing nerve fibre densities that are early events in people with diabetes [30].

We also observed correlations between hot and cold temperature perception thresholds and total body fat percentage measured by dual energy x-ray absorptiometry (DXA), and with abdominal subcutaneous fat assessed using MRI. Surprisingly, the directions of these associations indicate that thermal sensitivity (or the ability to detect the stimulus with a small temperature change) increased as the proportion of body fat increased. Importantly, these associations were independent of sex and of age [31]. Temperature thresholds depend on the contact area between the skin and the warm or cool surface with greater sensitivity when more receptors are excited by a larger contact area [32]. It is possible that increased subcutaneous fat resulted in an increased area of contact between the surface of the thermal aesthesiometer and the big toe but it was not possible to adjust for the size of the big toe in this study. The changes in temperature perception thresholds may alternatively be associated with skin thickness and thermal conduction and/or nerve density and tissue blood flow but investigation of these factors was beyond the scope of the present study. Obesity is characterised by a low-grade chronic inflammatory state, which is known to modulate pain, and people with chronic pain tend to have a greater total fat mass [33]. Thus it is possible that the increase in temperature sensitivity with increasing body fat percentage that we observe in our cohort may to be due to a change in small sensory nerve fibre function modulated by an increased release of pro-inflammatory cytokines or adipokines from adipose tissue.

The current study extends previous data suggesting that the functions of both small and large sensory fibres are impaired in neurologically asymptomatic individuals both with and without diabetes. The temperature perception thresholds to hot and cold stimuli associated with activation of small sensory afferent A-delta and C-nerve fibres that we measured at the big toe were strongly correlated with large sensory nerve fibre VPT measured at $125 \mathrm{~Hz}$ [8]. Large-fibre nerve dysfunction measured by VPT predicts foot ulceration, lower limb amputation and mortality [26]. However, to what extent assessment of small fibre function using QST may represent an additional screening tool to monitor disease progression and therapeutic impact remains uncertain [34].

\section{Limitations}

QSTs are psychophysical tests and their use as a diagnostic tool alone or in combination with other more direct tests is much debated [34]. We cannot overlook the fact that there are a number of factors that may affect temperature perception thresholds which we have been unable to measure, including alertness and attention, integration of sensory information from the stimulated skin area and skin thickness [25]. Our results provided no indication of an improvement with the intervention (Table 2). A retrospective power calculation based on a sample size of $n=90$ with $n=44$ in the treatment arm of the trial shows that we had $84 \%$ power to detect a 
$3.5^{\circ} \mathrm{C}$ temperature difference in HPT between baseline and the end of study with DHA+EPA treatment. That we observed only a $1^{\circ} \mathrm{C}$ mean temperature difference in HPT between baseline and end of study in the treatment arm, strongly suggests that there was no effect of the intervention on this variable but we would have needed a ten times bigger sample size to prove there was no effect of treatment. Additionally, with the very low CV $(\sim 6 \%)$ of the temperature perception threshold measurement techniques, this gives us further confidence in the validity of the findings. It is interesting to note that there was no worsening of temperature perception thresholds in the placebo arm of the trial. This finding is consistent with both the results of our previous study investigating the effect of the DHA+EPA intervention on VPTs as a measure of large sensory nerve fibre function [8] and the results of others [35]; and it is plausible that the duration of our trial (a minimum of 15 months and a maximum of 18 month) was insufficient for us to demonstrate a deterioration in temperature perception thresholds. Finally, we cannot be sure of the effect of duration of diabetes on temperature perception in our cohort, as identification of the date of onset of type 2 diabetes is often imprecise in clinical practice.

\section{Conclusions}

In summary, treatment with the highest licensed dose of DHA+EPA (as Omacor/Lovaza) did not modify small nerve fibre-mediated temperature perception in patients with NAFLD both with and without diabetes. In this high-risk patient group, without evidence of overt peripheral neuropathy, percentage body fat was independently associated with HPT and CPT. Both HPT and CPT were more strongly associated with the measure of overall body fatness than with any metabolic risk factors.

Acknowledgements The authors thank G. Wise, S. Triggs and B. Clancy (Research Nurses, National Institute for Health Research Southampton Biomedical Research Centre, Southampton, UK) for their support with the trial; L. England (Human Development and Health Academic Unit, Faculty of Medicine, University of Southampton, Southampton, UK) for research governance administration; and C. Gu and Y. Ye (Postgraduate researchers, Institute of Sound and Vibration Research, University of Southampton, Southampton, UK) for the thermal threshold perception protocol

Funding DHA+EPA (Omacor/Lovaza) and placebo were provided by Pronova Biopharma through Abbott Laboratories, Southampton, UK. This work was supported by the National Institute for Health Research through the NIHR Southampton Biomedical Research Unit grant and by a Diabetes UK allied health research training fellowship awarded to $\mathrm{KMcC}$ (Diabetes UK. BDA 09/0003937). CDB, PCC and ES are supported in part by the National Institute for Health Research Southampton Biomedical Research Centre.

Duality of interest PCC serves on the Clinical Advisory Board of Pronova Biopharma and has acted as a consultant to Amarin. All other authors declare that there is no duality of interest associated with their contribution to this manuscript.

Contribution statement All the named authors fulfil all three International Committee of Medical Journal Editors (ICMJE) uniform requirements for authorship of this manuscript. $\mathrm{KGMcC}, \mathrm{CDB}, \mathrm{GFC}$ and MJG made a substantial contribution to the conception and design of the study. KGMcC, ES and LB made a substantial contribution to the acquisition of the data. $\mathrm{CDB}, \mathrm{GFC}, \mathrm{KGMcC}, \mathrm{PCC}$ and $\mathrm{MJG}$ conducted the analysis and interpretation of data. $\mathrm{CDB}$ and GFC drafted the manuscript, PCC, MJG, ES, LB and KGMcC helped to revise it critically for important intellectual content. All the contributing authors approved the final version to be published. CDB is responsible for the integrity of the work as a whole and is the guarantor of this work.

\section{References}

1. Karvestedt L, Martensson E, Grill V et al (2011) The prevalence of peripheral neuropathy in a population-based study of patients with type 2 diabetes in Sweden. J Diabet Complications 25:97-106

2. Tesfaye S, Boulton AJ, Dickenson AH (2013) Mechanisms and management of diabetic painful distal symmetrical polyneuropathy. Diabetes Care 36:2456-2465

3. Schreiber AK, Nones CF, Reis RC, Chichorro JG, Cunha JM (2015) Diabetic neuropathic pain: physiopathology and treatment. World J Diabetes 6:432-444

4. Karvestedt L, Martensson E, Grill V et al (2009) Peripheral sensory neuropathy associates with micro- or macroangiopathy: results from a population-based study of type 2 diabetic patients in Sweden. Diabetes Care 32:317-322

5. Tesfaye S, Selvarajah D (2012) Advances in the epidemiology, pathogenesis and management of diabetic peripheral neuropathy. Diabetes Metab Res Rev 28(Suppl 1):S8-S14

6. Krumova EK, Geber C, Westermann A, Maier C (2012) Neuropathic pain: is quantitative sensory testing helpful? Curr Diab Rep 12:393-402

7. Korei AE, Istenes I, Papanas N, Kempler P (2016) Small-fiber neuropathy: a diabetic microvascular complication of special clinical, diagnostic, and prognostic importance. Angiology 67:49-57

8. McCormick KG, Scorletti E, Bhatia L et al (2015) Impact of high dose n-3 polyunsaturated fatty acid treatment on measures of microvascular function and vibration perception in non-alcoholic fatty liver disease: results from the randomised WELCOME trial. Diabetologia 58:1916-1925

9. Chao CC, Hsieh SC, Yang WS et al (2007) Glycemic control is related to the severity of impaired thermal sensations in type 2 diabetes. Diabetes Metab Res Rev 23:612-620

10. Shukla G, Bhatia M, Behari M (2005) Quantitative thermal sensory testing - value of testing for both cold and warm sensation detection in evaluation of small fiber neuropathy. Clin Neurol Neurosurg 107:486-490

11. Hafner J, Lee G, Joester J et al (2015) Thermal quantitative sensory testing: a study of 101 control subjects. J Clin Neurosci 22:588-591

12. Heldestad V, Linder J, Sellersjo L, Nordh E (2010) Reproducibility and influence of test modality order on thermal perception and 
thermal pain thresholds in quantitative sensory testing. Clin Neurophysiol 121:1878-1885

13. Moloney NA, Hall TM, Doody CM (2012) Reliability of thermal quantitative sensory testing: a systematic review. J Rehabil Res Dev 49:191-207

14. Yuen KC, Baker NR, Rayman G (2002) Treatment of chronic painful diabetic neuropathy with isosorbide dinitrate spray: a doubleblind placebo-controlled cross-over study. Diabetes Care 25:16991703

15. Patel N, Mishra V, Patel P, Dikshit RK (2014) A study of the use of carbamazepine, pregabalin and alpha lipoic acid in patients of diabetic neuropathy. J Diabetes Metab Disord 13:62

16. Zilliox L, Russell JW (2011) Treatment of diabetic sensory polyneuropathy. Curr Treat Options Neurol 13:143-159

17. Okuda Y, Mizutani M, Ogawa M et al (1996) Long-term effects of eicosapentaenoic acid on diabetic peripheral neuropathy and serum lipids in patients with type II diabetes mellitus. J Diabet Complications 10:280-287

18. Mori TA, Watts GF, Burke V, Hilme E, Puddey IB, Beilin LJ (2000) Differential effects of eicosapentaenoic acid and docosahexaenoic acid on vascular reactivity of the forearm microcirculation in hyperlipidemic, overweight men. Circulation 102:1264-1269

19. Coste TC, Gerbi A, Vague P, Pieroni G, Raccah D (2003) Neuroprotective effect of docosahexaenoic acid-enriched phospholipids in experimental diabetic neuropathy. Diabetes 52:2578-2585

20. Scorletti E, Bhatia L, McCormick KG et al (2014) Design and rationale of the WELCOME trial: a randomised, placebo controlled study to test the efficacy of purified long chain omega- 3 fatty treatment in non-alcoholic fatty liver disease. Contemp Clin Trials 37: 301-311

21. Scorletti E, Bhatia L, McCormick KG, et al (2014) Effects of purified eicosapentaenoic and docosahexaenoic acids in non-alcoholic fatty liver disease: results from the Welcome* study. Hepatology 60:1211-1221

22. Spruce MC, Bowling FL (2012) Diabetic foot screening: new technology versus $10 \mathrm{~g}$ monofilament. Int $\mathrm{J}$ Low Extrem Wounds 11: 43-48

23. Seah SA, Griffin MJ (2008) Normal values for thermotactile and vibrotactile thresholds in males and females. Int Arch Occup Environ Health 81:535-543
24. Nilsson T, Lundstrom R (2001) Quantitative thermal perception thresholds relative to exposure to vibration. Occup Environ Med 58:472-478

25. Kuhtz-Buschbeck JP, Andresen W, Gobel S, Gilster R, Stick C (2010) Thermoreception and nociception of the skin: a classic paper of Bessou and Perl and analyses of thermal sensitivity during a student laboratory exercise. Adv Physiol Educ 34:25-34

26. Miscio G, Guastamacchia G, Brunani A, Priano L, Baudo S, Mauro A (2005) Obesity and peripheral neuropathy risk: a dangerous liaison. J Peripher Nerv Syst 10:354-358

27. Malik RA, Veves A, Walker D et al (2001) Sural nerve fibre pathology in diabetic patients with mild neuropathy: relationship to pain, quantitative sensory testing and peripheral nerve electrophysiology. Acta Neuropathol 101:367-374

28. Dziemidok P, Szczesniak G, Kostrzewa-Zablocka E, Paprzycki P, Korzon-Burakowska A (2012) Current glycaemic control has no impact on the advancement of diabetic neuropathy. Ann Agric Environ Med 19:742-745

29. Crawford F, Inkster M, Kleijnen J, Fahey T (2007) Predicting foot ulcers in patients with diabetes: a systematic review and metaanalysis. QJM 100:65-86

30. Sumner CJ, Sheth S, Griffin JW, Cornblath DR, Polydefkis M (2003) The spectrum of neuropathy in diabetes and impaired glucose tolerance. Neurology 60:108-111

31. Hagander LG, Midani HA, Kuskowski MA, Parry GJ (2000) Quantitative sensory testing: effect of site and pressure on vibration thresholds. Clin Neurophysiol 111:1066-1069

32. Seah SA, Griffin MJ (2010) Thermotactile thresholds at the fingertip: effect of contact area and contact location. Somatosens Mot Res 27:82-92

33. Okifuji A, Hare BD (2015) The association between chronic pain and obesity. J Pain Res 8:399-408

34. Backonja MM, Walk D, Edwards RR et al (2009) Quantitative sensory testing in measurement of neuropathic pain phenomena and other sensory abnormalities. Clin J Pain 25:641-647

35. Polydefkis M, Arezzo J, Nash M et al (2015) Safety and efficacy of ranirestat in patients with mild-to-moderate diabetic sensorimotor polyneuropathy. J Peripher Nerv Syst 20:363-371

36. Guha IN, Parkes J, Roderick P et al (2008) Noninvasive markers of fibrosis in nonalcoholic fatty liver disease: validating the European Liver Fibrosis Panel and exploring simple markers. Hepatology 47: $455-460$ 